\title{
OS REGISTROS ELETRÔNICOS EM SAÚDE E SEUS POTENCIAIS IMPACTOS NO CAMPO DA SAÚDE PÚBLICA
}

\author{
Electronic Health Records and their Potential Impacts on the Public Health Field
}

\section{Luciana Tricai Cavalini'; Nathália Cristina Laurindo de Oliveira Ahiadzro²; Timothy Wayne Cook ${ }^{3}$}

Resumo Os Registros Eletrônicos em Saúde (RES) têm sido incorporados de forma crescente aos serviços de saúde, em escala global. O uso de dados provenientes de RES para a pesquisa e a prática em saúde pública ainda é incipiente, em comparação com os métodos tradicionais de coleta e processamento de dados, através de inquéritos e notificação de casos. Existe um potencial ainda inexplorado de adoção dos RES como um instrumento metodológico para o campo da saúde pública, o que depende da superação de diversos desafios tecnológicos sendo atualmente enfrentados pela área de informática em saúde. O presente artigo tem como objetivo realizar uma revisão crítica sobre tema dos RES e seu potencial impacto no campo da saúde pública, incluindo uma descrição das dimensões e processos relacionados aos desafios a serem enfrentados para adoção efetiva dos RES como um instrumento válido para uso na pesquisa e na prática em saúde pública. As soluções disponíveis para tais desafios são apresentadas, com foco nos padrões da área de informática em saúde que se relacionam aos requisitos de informação em saúde específicos do campo da saúde pública.

Palavras-chave: Registros Eletrônicos de Saúde, Informática em Saúde Pública, Vigilância em Saúde Pública

\begin{abstract}
Electronic Health Records (EHR) have been increasingly incorporated to healthcare services on a global scale. Using data from EHRs for the research and the practice in public health is still incipient, as compared to traditional methods of data collection and processing through surveys and case reporting. There is an untapped potential for the adoption of EHRs as a methodological tool in the public health field, which depends on the overcoming of several technological challenges currently being faced by the field of health informatics. This paper aims to conduct a critical review on the theme of EHRs and their potential impact on public health, including a description of the dimensions and processes related to the challenges to be faced for the effective application of EHRs as valid instruments for use in public health research and practice. Available solutions for such challenges are presented, focusing on healthcare informatics standards that are associated to the specific requirements health information for the public health field.
\end{abstract}

Keywords: Electronic Health Records, Public Health Informatics, Public Health Surveillance

\footnotetext{
1. Professora Adjunta do Departamento de Tecnologias da Informação e Educação em Saúde - Faculdade de Ciências Médicas - Universidade do Estado do Rio de Janeiro - Doutorado em Saúde Coletiva; 2. Bolsista do Programa de Apoio Técnico às Atividades de Ensino, Pesquisa e Extensão (PROATEC) - Universidade do Estado do Rio de Janeiro - Graduação em Odontologia; 3. Chief Technological Officer da MedWeb 3.0 - MSc in Health Informatics
} 


\section{Introdução}

Um Registro Eletrônico em Saúde (RES), aqui entendido como uma coleção longitudinal de informações em saúde no formato eletrônico, tem a função de armazenar no referido formato os dados coletados em uma ou mais consultas de um paciente, ou em qualquer outro contato entre um cidadão e um serviço de saúde". Tais registros apresentam a utilidade potencial de organização do fluxo de trabalho dos serviços de saúde, provimento de apoio à decisão diagnóstica e terapêutica, detecção de condições de saúde de interesse em estágios precoces, provimento de ferramentas de análise da qualidade e da produtividade dos serviços de saúde, geração de evidências para a tomada de decisão sobre as políticas setoriais, entre outras ${ }^{2}$.

Os RES têm sido propostos e desenvolvidos nos Estados Unidos e Europa Ocidental desde a década de $1960^{3}$. Há grande variabilidade no resultado final das implementações de tais registros, sejam os frutos de pesquisa acadêmica ou produtos da indústria de software ${ }^{4}$, mas alguns componentes básicos em comum podem ser identificados. Destacam-se os seguintes:

Componente Administrativo: em termos gerais, engloba necessariamente um conjunto de informações sobre Identificação do Paciente (dados demográficos tais como nome, sexo, idade e endereço), Dados de Admissão (data, hora, local ou serviço, queixa primária) e Dados de Alta (data, hora, diagnóstico de alta, condição da alta, dados de óbito ou transferência) $)^{5}$.

Componente de Laboratório: coleta de dados sobre o pedido do exame (solicitante, material, tipo de exame), o resultado do exame (laudo) e dados administrativos (codificação do procedimento, custo do exame, faturamento) ${ }^{6}$.

Componente de Radiologia: coleta igualmente dados sobre o pedido, o resultado e aspectos administrativos.
Tem como característica específica a utilização de um formato padrão para a digitalização das imagens radiográficas (DICOM) ${ }^{7}$.

Componente de Farmácia: contempla dados administrativos relacionados ao gerenciamento dos estoques farmacêuticos, mas seu principal componente é o Sistema Computadorizado de Prescrição Eletrônica $(\mathrm{SCPE})^{8}$. Um SCPE contempla uma estruturação do processo de emissão de uma prescrição medicamentosa, com dados sobre o nome da droga, dosagem, via e frequência de administração, registrada pelo prescritor através de um formulário eletrônico estruturado. A incorporação desta tecnologia evita, no mínimo, as interpretações incorretas de uma prescrição médica em função de problemas de legibilidade, comuns em prescrições manuscritas 9 . SCPEs podem contemplar utilidades adicionais, tais como sistemas de apoio à prescrição de complexidade crescente, desde algoritmos simples de adequação da dosagem de uma substância específica ao que é recomendado em termos de eficácia e segurança, até ferramentas mais complexas que sugerem uma gama de medicamentos em função de um diagnóstico informado ${ }^{10}$.

Componente de Documentação Clínica: este é o componente mais vasto e complexo, e o menos desenvolvido dos RES. A documentação clínica diz respeito a toda informação clinicamente relevante levantada pelo profissional de saúde em uma consulta. Diz respeito não apenas à consulta médica, mas a toda a amplitude de serviços executados por profissionais de saúde: consultas de enfermagem, imunizações, procedimentos cirúrgicos, curativos, órteses e próteses, atendimentos odontológicos, de reabilitação, entre outros ${ }^{11}$.

Até o presente momento, pode-se considerar que o uso dos RES no campo da saúde pública, tanto na área de pesquisa científica quanto na vigilância em saúde, 
é limitado; entretanto, as potencialidades para a sua adoção estão presentes. Este estudo tem como objetivo realizar uma revisão crítica sobre tema dos RES e seu potencial impacto no campo da saúde pública, incluindo uma descrição das dimensões e processos relacionados aos desafios a serem enfrentados para adoção efetiva dos RES como um instrumento válido para uso na pesquisa e na prática em saúde pública.

\section{Métodos}

O presente estudo realizou uma revisão narrativa da literatura científica relacionada à interface entre os campos da saúde pública e da informática em saúde, com foco no papel dos RES. Os resultados desta revisão são apresentados em duas seções, que apresentam os atuais desafios para implementação dos RES nos serviços de saúde, dimensionados de acordo com aspectos financeiros, culturais e tecnológicos, assim como as soluções atualmente existentes para a superação de tais desafios, com foco nos padrões da área de informática em saúde. A discussão do artigo estabelece a interface entre o cenário obtido a partir dos resultados da revisão e as aplicações de RES no campo da saúde pública, tanto na área de pesquisa quanto nas ações de vigilância em saúde.

Justifica-se a realização de uma revisão narrativa em relação a este tema, visto que, especialmente no âmbito da produção científica nacional, a integração dos campos da saúde pública e da informática em saúde ainda pode ser considerada uma inovação, especialmente no que tange à apreciação crítica e reflexiva sobre o papel dos RES na interface entre ambos os campos.

\section{Resultados}

A literatura científica da área de informática em saúde tem apresentado um número crescente de publi- cações relacionadas aos desafios econômicos, culturais e tecnológicos relacionados à implementação dos RES na prática dos serviços de saúde. Ao mesmo tempo, expande-se a produção científica relativa ao desenvolvimento de soluções que superem tais desafios, especialmente no que tange à definição de padrões para os RES. As principais questões relacionadas a ambos os temas são apresentadas a seguir.

\section{Desafios na Implementação de RES}

Embora seja reconhecido o potencial de aprimoramento da qualidade dos serviços de saúde pela implementação em escala crescente de RES, o que pode ser verificado pelas quantidades massivas de investimentos para a incorporação de recursos de tecnologia da informação em nível local, nacional e global, o fato é que o setor saúde ainda é um dos menos informatizados da sociedade ${ }^{12}$. O descompasso entre seu alegado potencial e a efetiva implementação de RES pode ser analisado em função de três dimensões:

A Dimensão Financeira: Os RES são as peças de software de mais alto custo no mercado. Há também grandes custos indiretos, tais como aquisição de hardware e treinamento de usuários. Entretanto, o custo não deve ser analisado de forma isolada, mas sim em termos de custo-efetividade real ou percebida ${ }^{13}$. Neste caso, criase um significativo obstáculo à adoção de RES, no que tange as duas dimensões restantes:

A Dimensão Cultural: Processos de gerenciamento de mudanças no fluxo de trabalho de instituições com grande complexidade de rede e estrutura hierárquica são lentos, dispendiosos e requerem grande empenho políticos dos atores envolvidos. A transição de um serviço de saúde dos registros em papel para os RES é um processo desta natureza ${ }^{14}$ 
A Dimensão Tecnológica: Embora em termos de hardware as questões tecnológicas relativas à implementação de um RES sejam de menor magnitude, as questões tecnológicas relacionadas ao software ainda são um desafio. Em outros setores da economia, a informatização tem avançado mais rapidamente do que na saúde, e uma das razões para que isto aconteça é que o software necessário para os processos destes outros setores (ex: bancário) é incomparavelmente mais simples do que o requerido pelo setor saúde ${ }^{15}$. Dois aspectos desta complexidade devem ser destacados:

A Dinâmica Espacial e Temporal: O sistema de saúde pode ser definido como um sistema dinâmico. Neste caso em particular, a dinâmica espacial refere-se ao fato de que os requisitos de um sistema podem ser extremamente diferentes, mesmo com grande proximidade geográfica (por exemplo, uma Unidade de Saúde da Família e uma Unidade de Pronto Atendimento no mesmo bairro) ${ }^{16}$. A dinâmica temporal diz respeito à velocidade crescente com que a incorporação tecnológica médico-hospitalar tem criado novos conceitos, alterado ou mesmo tornado obsoletos conceitos já estabelecidos ${ }^{17}$.

A Complexidade do Domínio: Os serviços de saúde se estruturam em um sistema complexo, havendo uma estimativa de que o domínio da saúde seja composto por aproximadamente trezentos mil conceitos, que apresentam correlações complexas entre si, e não há fácil consenso entre especialistas sobre a definição e os componentes destes conceitos. Isto é uma razão pela qual há uma baixa proporção de elaboração e adoção de protocolos clínicos ${ }^{18}$.

Em função desta complexidade espaço-temporal e de domínio, RES apresentam um risco significativo de não atender aos requisitos do cliente, em função da dificuldade de elaboração de um modelo de dados e uma interface de usuário correspondente que satisfaça todos os usuários finais do aplicativo ${ }^{19}$. Além disso, os custos de manutenção de um RES podem ser impeditivos em face de sua percebida efetividade, visto que, a cada modificação de um conceito que implique em mudança do modelo de dados, de acordo com a modelagem de software mais prevalente, há a necessidade de refatoração dos mecanismos de persistência (por exemplo, a base de dados) e das interfaces de usuário, assim como subsequente reimplementação, reteste e redistribuição do aplicativo, todas tarefas intensivas em termos de dispêndio de tempo e recursos. A alternativa é a relativamente rápida obsolescência do aplicativo, e a aquisição de um novo, com novos (e altos) custos de implementação ${ }^{20}$.

\section{Padrões para os RES}

Em face da constatação destes desafios, diversas instituições em âmbito internacional têm realizado consideráveis esforços no sentido da elaboração de padrões para os $\mathrm{RES}^{21}$. Os padrões sendo elaborados podem ser agrupados em duas categorias: Especificações e Terminologias.

As Especificações são documentos que definem os procedimentos para a elaboração de RES, e podem dizer respeito tanto aos aspectos técnicos (mais ou menos detalhados) quanto aos aspectos clínicos (onde se destacam os modelos de representação do conhecimento). Algumas especificações têm sido adotadas como normas nacionais ou internacionais para a área de informática em saúde ${ }^{22}$.

A International Standards Organization (ISO) criou em 2003 um Comitê Técnico para a área de informática em saúde, o TC 21523. Desde então, vários componentes (modificados ou não) de duas importantes especificações têm sido convertidos em Normas ISO, a saber:

Health Level 7 (HL7): As especificações HL7 têm sido adotadas como padrão de troca de mensagens entre 
diferentes RES desde a publicação do Health Informatics Portability and Accountability Act (HIPAA), em 1996, nos Estados Unidos ${ }^{24}$. Suas versões HL7v2.x foram largamente implementadas em sistemas laboratoriais e radiológicos norte-americanos, e a versão HL7v3 especifica um Modelo de Informação de Referência (Reference Information Model - RIM) para a troca de mensagens sobre documentação clínica dos RES ${ }^{25}$. Os tipos de dados HL7 foram convertidos na Norma ISO $21090^{26}$.

Modelagem Multinível: As especificações openEHR originalmente propuseram um método não convencional de modelagem de RES, denominado "modelo dual" ou "modelo multinível", no qual a definição dos mecanismos de persistência é orientado por um conjunto de classes genéricas, necessárias e suficientes para a persistência de qualquer dado em saúde, assim como o relacionamento de herança destas classes, seus atributos e métodos. Este conjunto é denominado Modelo de Referência ${ }^{27}$.

A característica genérica das classes do Modelo de Referência não é suficiente para definir o que no modelo relacional é conhecido como "restrições de integridade". Por isso, as especificações openEHR definem um Modelo de Domínio, separado do Modelo de Referência, no qual os conceitos são modelados pela definição de restrições (combinação e restrição de atributos) das classes do Modelo de Referência. Assim, cada conceito em saúde é modelado através do Modelo de Domínio, e as definições de restrições às classes do Modelo de Referência são expressas em artefatos computacionais denominados "arquétipos"28.

Os arquétipos provêm a interpretação semântica dos objetos persistidos de acordo com o Modelo de Referência. Desta forma, as especificações openEHR têm a meta de desenvolvimento de um RES centrado no paciente e dotado de interoperabilidade no nível semântico. Conceitos provenientes das especificações
openEHR podem ser identificados nas Normas ISO 20514, 18308 e, principalmente, na série $13606^{29}$.

A mais recente evolução das especificações openEHR, no sentido de facilitar a implementação, na prática dos serviços de saúde, de sistemas de informação em saúde interoperáveis baseados em modelagem multinível, são as especificações Multilevel Healthcare Information Modeling (MLHIM), baseadas em tecnologias web semânticas tais como extensible Markup Language (XML), Resource Description Framework (RDF) e Web Ontology Language $(\mathrm{OWL})^{30}$.

Além das especificações, há um esforço intensivo relacionado à elaboração, desenvolvimento e harmonização de terminologias em saúde, que podem ser entendidas como um sistema estruturado e multiaxial de vocabulários controlados para um domínio específico (no caso, o da saúde) ${ }^{31}$. Embora a ISO tenha envidado esforços no sentido de normalização do desenvolvimento de terminologias de saúde, a principal instituição internacional envolvida nesta iniciativa é a International Health Terminology Standards Organization (IHTSDO). A IHTSDO é a responsável pela manutenção da Systematic Nomenclature of Medical Terms (SNOMED), a maior e mais abrangente terminologia médica, originalmente elaborada pelo Colégio Americano de Patologistas. A IHTSDO realiza importantes projetos de harmonização da SNOMED com outras terminologias (tais como a Logical Observational Identifiers, Names and Codes - LOINC), vocabulários controlados (tais como o Medical Subject Headings - MeSH) e classificações (tais como a Classificação Internacional de Doenças - CID) $)^{32}$.

Espera-se que estes massivos esforços institucionais globais e a intensificação da pesquisa acadêmica e industrial na área de informática em saúde produzam a inovação tecnológica necessária para a superação dos atuais desafios à implementação de larga escala de RES. Em particular, o campo de saber da saúde pública se beneficiaria grandemente da realização dos RES na 
sociedade, tanto em termos acadêmicos quanto na prática dos serviços de saúde ${ }^{33}$.

\section{Discussão}

Há uma potencialidade inerente ao uso de RES em saúde pública, tanto na área acadêmica quanto de vigilância em saúde. Em termos de pesquisa científica, os RES podem promover os seguintes aprimoramentos:

Produção de Evidências: Um dos grandes desafios atuais da saúde pública é a produção de evidências científicas variadas e em quantidade considerável. Não obstante o enorme crescimento da área desde a segunda metade do século 20 , ainda há um grande número de investigações epidemiológicas a serem realizadas (em termos causais e também na avaliação de intervenções e estudos prognósticos) ${ }^{34}$. Os estudos epidemiológicos são em geral lentos e custosos, pois frequentemente requerem a recoleta de informações que foram previamente obtidas pelos serviços de saúde, mas não adequadamente registradas ${ }^{35}$. O acesso a RES de boa cobertura e qualidade aumentariam a gama de condições de saúde que poderiam ser estudadas, em princípio com custos já parcialmente absorvidos pela implementação prévia do RES ${ }^{36}$.

Precisão: A precisão das estimativas de parâmetros de estudos epidemiológicos é determinada por duas variáveis: a variabilidade das estimativas do parâmetro na população de referência (que o pesquisador não pode controlar) e o tamanho da amostra do estudo (que o pesquisador pode controlar), sendo que, quanto maior a amostra analisada, maior a precisão da estimativa. A disponibilidade de informações provenientes de RES tem o potencial de realização de estudos (especialmente os de caráter retrospectivo) com maiores amostras ${ }^{37}$.
Validade: a validade de um estudo epidemiológico diz respeito ao controle de vieses e confundimento. Em relação aos vieses, o acesso a grandes bases de dados provenientes de Registros Eletrônicos em Saúde, especialmente no caso de estudos retrospectivos, é capaz de prover o acesso aos fatores de exposição e outras covariáveis com um mínimo de intervenção do responsável pelo registro das informações que, por ter coletado a informação no ponto e no momento de cuidado, tem menor probabilidade de ter sido "enviesado" na sua investigação por um estudo que seria realizado no futuro e provavelmente sem seu conhecimento ${ }^{38}$. Mesmo para estudos prospectivos, a informatização dos registros facilita o estabelecimento de controles sobre a investigação, tais como protocolos uniformizados de registro da investigação, cegamento do entrevistador, alocação aleatória do controle por placebo, entre outros ${ }^{39}$.

Confiabilidade: A Confiabilidade de uma medida diz respeito à sua capacidade de representar a realidade de forma consistente e independente do tempo ou do observador. A implementação de RES tem um potencial (dependente de outras variáveis associadas ao processo) de melhorar a confiabilidade das informações em saúde, pela maior estruturação dos métodos de registro e dos próprios fluxos de trabalho nas instituições de saúde ${ }^{40}$.

Em relação à prática da saúde pública nos serviços de saúde, pode-se destacar dois processos de trabalho que poderiam ser beneficiados pela implementação de RES: a utilização do método epidemiológico para a avaliação dos serviços de saúde e a vigilância em saúde ${ }^{41}$. Considerando-se que as principais questões relacionadas ao método epidemiológico já foram cobertas na seção anterior sobre a pesquisa acadêmica em saúde pública, o enfoque a seguir será dado sobre o processo de trabalho da vigilância em saúde. 
A vigilância em saúde é o processo de trabalho em saúde que se propõe a identificar a ocorrência de fenômenos de saúde nas comunidades, com o intuito de controlá-los e, sempre que possível, preveni-los. A vigilância em saúde se baseia no princípio da "informação para a ação". Ou seja, as ações de prevenção e controle de doenças e outros agravos em saúde são desencadeados pelas informações obtidas sobre a ocorrência destes eventos. Para serem efetivas, as informações obtidas pela vigilância em saúde requerem quatro características: oportunidade, atualidade, disponibilidade e cobertura ${ }^{42}$. A implementação dos RES poderia contribuir para a qualificação destes quatro processos da seguinte forma:

Incremento da Oportunidade: RES com sistemas de detecção de doenças de notificação compulsória tornam o processo de vigilância em saúde independente da espera por notificação passiva dos profissionais de saúde (que é, reconhecidamente, bastante omitida) ou da busca ativa dos sanitaristas nos serviços. Com isso, agregados inusitados de doenças de interesse para a vigilância em saúde podem ser detectados em estágios mais precoces do que através da notificação tradicional ${ }^{43}$.

Maior Atualidade: caso os RES realizem a coleta dos dados no momento e no local de cuidado, é possível obter relatórios atualizados de atendimento dentro da frequência desejada, evitando as digitações secundárias recorrentes nos sistemas de informação atuais ${ }^{44}$.

Maior Disponibilidade: do mesmo modo que foi descrito para a pesquisa epidemiológica acadêmica, grandes massas de dados provenientes de RES amplamente implementados e integrados ou, preferivelmente, interoperáveis, proveriam os serviços de vigilância em saúde de uma maior quantidade de registros individuais que poderiam ser acessados por rotinas de busca predefinidas ${ }^{45}$.
Aumento da Cobertura: a cobertura da vigilância em saúde, em um cenário de implementação de RES, dependeria, evidentemente, da cobertura de implementação dos próprios registros. Entretanto, em serviços não dotados de estrutura de vigilância em saúde, os RES poderiam ser verificados remotamente para a detecção de condições de interesse $e^{46}$.

\section{Conclusões}

No campo da saúde pública ainda é, apesar da crescente produção científica relacionada, amplamente inexplorado. Uma das maiores barreiras para a sua expansão é a limitação de acesso a informações precisas, fidedignas e oportunas, que promovam a geração de novas evidências científicas e permitam um controle e prevenção mais efetivo das doenças de interesse comunitário ${ }^{47}$. O potencial de contribuição dos RES para o aprimoramento da qualidade dos serviços prestados pelo sistema de saúde é reconhecido, e há grande expectativa em relação à sua concretização no futuro. Resta aos pesquisadores da área manterem seu empenho na superação dos desafios tecnológicos que ainda persistem ${ }^{48}$.

O horizonte de superação das barreiras tecnológicas para uma implementação ampliada e significativa dos RES nos sistemas de saúde globais é visível, em função dos recentes desenvolvimentos relacionados aos padrões da área de informática em saúde. Espera-se, com isso, a geração de informações válidas e confiáveis através dos RES, que possam ser, assim, plenamente incorporados à pesquisa e à prática no campo da saúde pública, num futuro próximo.

\section{Referências}

1. GunterTD, Terry NP. the emergence of national electronic health record architectures in the United States and Australia: models, costs, and questions. J Med Internet Res 2005; 7: e3. 
2. Häyrinen K, Saranto K, Nykänen P. Definition, structure, content, use and impacts of electronichealthrecords: a review of the research literature. Int J Med Inform 2008; 77: 291-304.

3. Grimson J. Delivering the electronic healthcare record for the 21st century. Int J Med Inform 2001; 64: 111-27.

4. Wu S, Chaudhry B, Wang J, Maglione M, Mojica W, Roth E, Morton SC, Shekelle PG. Impact of health information technology on quality, efficiency, and costs of medical care. Ann Intern Med 2006; 144: 742-52.

5. Bays RA, Kaelin LD. Electronic medical records for the office. J Vasc Surg 2010; 51: 1302-8.

6. Long RE, Smith A, Machotka SV, Chlipala E, Cann J, Knight B, Kawano Y, Ellin J, Lowe A. Scientific and Regulatory Policy Committee (SRPC) paper: validation of digital pathology systems in the regulated nonclinical environment. Toxicol Pathol 2013; 41: 115-24.

7. Soriano E, Plazzotta F, Campos F, Kaminker D, Cancio A, Aguilera Díaz J, Luna D, Seehaus A, Carcía Mónaco R, de Quirós FG. Integration of healthcare information: from enterprise PACS to patient centered multimedia health record. Stud Health Technol Inform 2010; 160: 126-30

8. Cufar A, Droljc A, Orel A. Electronic medication ordering with integrated drug database and clinical decision support system. Stud Health Technol Inform 2012; 180: 693-7

9. Rupp MT, Warholak TL. Evaluation of e-prescribing in community pharmacy: bestpractice recommendations. J Am Pharm Assoc 2008; 48: 364-70.

10. Chaffee BW. Future of clinical decision support in computerized prescriber order entry. Am J Health Syst Pharm 2010; 67: 932-5.

11. Vedvik E, Tjora AH, Faxvaag A. Beyond the EPR: complementary roles of the hospital-wide electronic health record and clinical departmental systems. BMC Med Inform Decis Mak 2009; 9: 29.

12. Rothschild AS, Dietrich L, Ball MJ, Wurtz H, Farish-Hunt H, Cortes-Comerer N. Leveraging systems thinking to design patient-centered clinical documentation systems. Int J Med Inform 2005; 74: 395-8.

13. Bassi J, Lau F. Measuring value for money: a scoping review on economic evaluation of health information systems. J Am Med Inform Assoc 2013; 20: 792-801.

14. Hannan T, Brooks P. Health workforce changes and the roles of information technology associated with these changes. Intern Med J 2012; 42: 722-7.

15. Zwaanswijk M, Verheij RA, Wiesman FJ, Friele RD. Benefits and problems of electronic information exchange as perceived by health care professionals: an interview study. BMC Health Serv Res 2011; 11: 256

16. Mi T, Rajasekaran S, Aseltine R. Efficient algorithms for fast integration on large data sets from multiple sources. BMC Med Inform Decis Mak 2012; 12: 59.

17. Albers DJ, Hripcsak G. Using time-delayed mutual information to discover and interpret temporal correlation structure in complex populations. Chaos 2012; 22: 013111.

18. Mason-Blakley F, Weber J. A systems theory classification of EMR hazards: preliminary results. Stud Health Technol Inform 2013; 183: 214-9.

19. Al-Azmi SF, Al-Enezi N, Chowdhury RI. Users' attitudes to an electronic medical record system and its correlates: a multivariate analysis. HIM J 2009; 38: 33-40.

20. Eastaugh SR. The total cost of EHR ownership. Healthc Financ Manage 2013; 67: 66-70.

21. Engel K, Blobel B, Pharow P. Standards for enabling health informatics interoperability. Stud Health Technol Inform 2006; 124: 145-50.

22. Blobel B. Advanced and secure architectural EHR approaches. Int J Med Inform 2006; 75: 185-90.

23. Klein GO. ISO and CEN standards for health informatics: synergy or competition. Stud Health Technol Inform 2003; 96: 259-65.
24. Amatayakul M. Help in setting access controls: using the HL7 RBAC Healthcare Permission Catalog to reduce data breaches. J AHIMA 2008; 79: 56-7, 61.

25. Namli T, Aluc G, Dogac A. An interoperability test framework for HL7-based systems. IEEE Trans Inf Technol Biomed 2009; 13: 389-99.

26. Sun S, Austin T, Kalra D. A data types profile suitable for use with ISO EN 13606. J Med Syst 2012; 36: 3621-35.

27. Chen R, Klein G. The openEHR Java reference implementation project. Stud Health Technol Inform 2007; 129: 58-62.

28. Garde S, Knaup P, Hovenga E, Heard S. Towards semantic interoperability for electronic health records. Methods Inf Med 2007; 46: 332-43.

29. Martínez-Costa C, Menárguez-Tortosa M, Fernández-Breis JT. Towards ISO 13606 and openEHR archetype-based semantic interoperability. Stud Health Technol Inform 2009; 150: 260-4.

30. Cavalini, L. T., Cook, T. W. Use of XML Schema Definition for the development of semantically interoperable healthcare applications. Lecture Notes in Computer Science 2014; 8315:125-145.

31. International Standards Organization. ISO/TS 17117:2002. Controlled health terminology: structure and high-level indicators. Geneva; 2002.

32. Campbell JR, Brear H, Scichilone R, White S, Giannangelo K, Carlsen B, Solbrig $\mathrm{H}$, Fung KW. semantic interoperation and electronic health records: context sensitive mapping from SNOMED CT to ICD-10. Stud Health Technol Inform 2013 192: 603-7.

33. Herasevich V, Pickering BW, Dong Y, Peters SG, Gajic O. Informatics infrastructure for syndrome surveillance, decision support, reporting, and modeling of critical illness. Mayo Clin Proc 2010; 85: 247-54

34. Grimshaw J, Eccles M, Tetroe J. Implementing clinical guidelines: current evidence and future implications. J Cont Educ Health Profess 2004; 24: S31-7.

35. Sloane PD, MacFarquhar JK, Sickbert-Bennett E, Mitchell CM, Akers R, Weber DJ, Howard K. Syndromic surveillance for emerging infections in office practice using billing data. Ann Fam Med 2006; 4: 351-8.

36. Fraser HS, Biondich P, Moodley D, Choi S, Mamlin BW, Szolovits P. Implementing electronic medical record systems in developing countries. Inform Prim Care 2005; 13: 83-95.

37. Wieland ML, Wu ST, Kaggal VC, Yawn BP. Tracking health disparities through natural-language processing. Am J Public Health 2013; 103: 448-9.

38. Bremner SA, Carey IM, DeWilde S, Richards N, Maier WC, Hilton SR, Strachan DP, Cook DG. Early-life exposure to antibacterials and the subsequent development of hayfever in childhood in the UK: case-control studies using the General Practice Research Database and the Doctors' Independent Network. Clin Exp Allergy 2003; 33: 1518-25.

39. Lau B, Gange SJ, Moore RD. Interval and clinical cohort studies: epidemiological issues. AIDS Res Hum Retroviruses 2007; 23: 769-76.

40. Biermans MC, Verheij RA, de Bakker DH, Zielhuis GA, de Vries Robbé PF. Estimating morbidity rates from electronic medical records in general practice. Evaluation of a grouping system. Methods Inf Med 2008; 47: 98-106.

41. Luchenski S, Balasanthiran A, Marston C, Sasaki K, Majeed A, Bell D, Reed JE. Survey of patient and public perceptions of electronic health records for healthcare, policy and research: study protocol. BMC Med Inform Decis Mak 2012; 12: 40.

42. Silva Júnior JB. Epidemiologia em serviço: uma avaliação de desempenho do Sistema Nacional de Vigilância em Saúde [tese de doutorado]. Campinas: Universidade Estadual de Campinas; 2004

43. Huaman MA, Araujo-Castillo RV, Soto G, Neyra JM, Quispe JA, Fernandez MF, Mundaca CC, Blazes DL. Impact of two interventions on timeliness and data quality of an electronic disease surveillance system in a resource limited setting (Peru): a prospective evaluation. BMC Med Inform Decis Mak 2009; 9: 16. 
44. Boehmer TK, Patnaik JL, Burnite SJ, Ghosh TS, Gershman K, Vogt RL. Use of hospital discharge data to evaluate notifiable disease reporting to Colorado's Electronic Disease Reporting System. Public Health Rep 2011; 126: 100-6.

45. Hay SI, George DB, Moyes CL, Brownstein JS. Big data opportunities for global infectious disease surveillance. PLoS Med 2013; 10: e1001413.

46. Lober WB, Baer A, Karras BT, Duchin JS. Collection and integration of clinical data for surveillance. Stud Health Technol Inform 2004; 107: 1211-5.
47. Azim T, Saidel TJ, Brown T. Essential elements of strategic information systems to guide focused HIV responses in south and south-east Asia. AIDS 2010; 24: S54-61.

48. López-Alonso V, Hermosilla-Gimeno I, López-Campos G, Mayer MA. Future challenges of biomedical informatics for translational medicine. Stud Health Technol Inform 2013; 192: 942 
